\title{
ADESÃO AO TRATAMENTO MEDICAMENTOSO POR PESSOAS COM TRANSTORNO DE ANSIEDADE*
}

\author{
Ligiane Paula da Cruz de Sousa ${ }^{1}$, Kelly Graziani Giacchero Vedana ${ }^{2}$, Adriana Inocenti Miasso ${ }^{2}$
}

\begin{abstract}
RESUMO: Este estudo verificou os fatores relacionados à adesão de pessoas com transtorno de ansiedade quanto à farmacoterapia prescrita. Estudo quantitativo, transversal, descritivo, realizado com 161 pessoas de um serviço de saúde mental, num município do estado de São Paulo, entre 01 de janeiro e 31 de dezembro de 2012. Utilizou-se revisão de prontuários, Teste de Medida de Adesão ao Tratamento, Inventário de Ansiedade de Beck e roteiro sociodemográfico. A maioria dos participantes foi considerada aderente aos medicamentos. Não foram evidenciadas diferenças estatisticamente significativas entre a adesão e as variáveis investigadas. Destacase a alta porcentagem de participantes em uso prolongado de benzodiazepínicos, com ansiedade moderada e severa apesar da adesão aos medicamentos e que desconheciam a dose dos medicamentos prescritos. Este estudo aponta elementos que interferem na segurança do tratamento farmacológico da pessoa com transtorno de ansiedade e que são passíveis de intervenção, contribuindo para a otimização do seu tratamento.

DESCRITORES: Transtornos de ansiedade; Adesão à medicação; Pacientes ambulatoriais.
\end{abstract}

\section{COMPLIANCE WITH MEDICATION TREATMENT BY PEOPLE WITH ANXIETY DISORDER}

ABSTRACT: In this study, the factors related to compliance with the prescribed drugs therapy are verified among people with anxiety disorder. Quantitative, cross-sectional, descriptive study, involving 161 patients from a mental health service in a city of the state of São Paulo, between January 01st and December 31st 2012. Patient histories were reviewed and the Treatment Adherence Measure, Beck Anxiety Inventory and a sociodemographic script were used. Most participants were considered compliant with the drugs. No statistically significant differences were evidenced between compliance and the research variables. The high percentage of participants using benzodiazepines over a long period is highlighted, suffering from moderate and severe anxiety despite the medication compliance and who were unaware of the prescribed medication dose. This study appoints elements that interfere in the safety of the drug treatment for people with anxiety disorder for whom intervention is possible, contributing to improve their treatment.

DESCRIPTORS: Anxiety disorders; Medication compliance; Outpatients.

\section{ADHESIÓN AL TRATAMIENTO MEDICAMENTOSO DE PERSONAS CON TRASTORNO DE ANSIEDAD}

RESUMEN: El estudio verificó factores relacionados a adhesión de personas con trastorno de ansiedad a la farmacoterapia prescripta. Estudio cuantitativo, transversal, descriptivo, realizado con 161 personas en servicio de salud mental de un municipio del Estado de São Paulo, del 01 de enero al 31 de diciembre de 2012. Se aplicó revisión de historias clínicas, Test de Medida de Adhesión al Tratamiento, Inventario de Ansiedad de Beck y relevamiento sociodemográfico. La mayoría de participantes calificó como adherente a la medicación. No se evidenciaron diferencias estadísticamente significativas entre adhesión y las variables investigadas. Se resalta el alto porcentaje de pacientes con uso prolongado de benzodiazepínicos, con ansiedad moderada y severa a pesar de adherir al tratamiento, y el desconocimiento de dosis de los medicamentos prescriptos. El estudio detecta factores que interfieren en la seguridad del tratamiento farmacológico del paciente con trastornos de ansiedad, pasibles de intervención, contribuyendo a su optimización..

DESCRIPTORES: Trastornos de Ansiedad; Cumplimiento de la Medicación; Pacientes Ambulatorios. *Artigo extraído da dissertação de mestrado intitulada: "Transtornos de Ansiedade: investigação da adesão à terapêutica
medicamentosa". Universidade de São Paulo, 2014.

${ }^{1}$ Enfermeira. Mestre em Enfermagem. Colégio Fundação Armando Alvares Penteado. Ribeirão Preto, SP, Brasil.

${ }^{2}$ Enfermeira. Doutora em Enfermagem. Docente de Enfermagem. Universidade de São Paulo. Ribeirão Preto, SP, Brasil.

Universidade de São Paulo

Av. Bandeirantes, 3900 - 14040-902 - Ribeirão Preto, SP, Brasil

E-mail: amiasso@eerp.usp.br 


\section{- INTRODUÇÃO}

Transtornos de ansiedade são considerados grande problema para a população mundial, estando associados a elevado sofrimento e limitações ${ }^{(1)}$. Estudo epidemiológico conduzido em cidades brasileiras identificou que os transtornos ansiosos foram os mais prevalentes $(17,6 \%)$ em relação aos demais transtornos mentais ${ }^{(2)}$.

Além de provocar sofrimento significativo, tais transtornos estão relacionados a elevados custos diretos e indiretos e estão entre os mais debilitantes transtornos psiquiátricos na vida adulta ${ }^{(3)}$, exigindo diagnóstico precoce e acompanhamento especializado ${ }^{(4)}$.

Existem medicamentos que podem ser utilizados para o tratamento dos transtornos de ansiedade, entre os quais destacam-se os antidepressivos e os benzodiazepínicos. A eficácia dos benzodiazepínicos é bem documentada nos tratamentos de curta duração, porém o uso prolongado é contraindicado devido aos riscos de efeitos adversos, incluindo a dependência( ${ }^{(5)}$.

Nos últimos anos, os antidepressivos, por serem bem tolerados e por não ocasionarem dependência, estabeleceram-se como tratamento de primeira linha para as diferentes categorias de transtorno de ansiedade(6). Entretanto, a adesão aos antidepressivos é desafiadora, pois aproximadamente metade dos pacientes interrompe o tratamento medicamentoso nos primeiros seis meses ${ }^{(7-9)}$. Pesquisa conduzida nos EUA revelou que $57 \%$ dos pacientes com diagnóstico de transtorno de ansiedade investigados eram não aderentes à terapia antidepressiva ${ }^{(10)}$.

Conceituar adesão ao tratamento consiste em tarefa complexa, pois não há consenso na literatura a esse respeito. Para a Organização Mundial de Saúde, a adesão pode ser definida como o grau em que o comportamento de uma pessoa coincide com as recomendações acordadas com um prestador de assistência sanitária ${ }^{(11)}$. Estudos defendem, todavia, a compreensão do paciente como sujeito ativo, que participa e assume responsabilidades no processo de aderir ou não ao tratamento ${ }^{(12)}$.

Constata-se que os transtornos de ansiedade apresentam prevalência significativa na sociedade, causam sofrimentos pessoais e encargos sociais e tendem a ser crônicos, se não tratados adequadamente. Deste modo, a adesão ao tratamento medicamentoso constitui temática de interesse para a literatura, por ser fator potencialmente modificável na busca de melhor prognóstico desses pacientes.

Todavia, recente revisão de literatura ${ }^{(13)}$ sobre a temática aponta que poucos estudos têm investigado preditores de não adesão ao tratamento medicamentoso em pacientes com transtornos de ansiedade, e que os estudos identificados são consistentes ao descrever alta taxa de não adesão nessa clientela, enfatizando a importância de mais pesquisas a esse respeito. Estudos apontam que a não adesão à terapêutica medicamentosa pode estar relacionada a uma complexidade de fatores ligados ao paciente (sexo, idade, etnia, estado civil, escolaridade e nível socioeconômico); ao regime terapêutico (tratamento complexo, efeitos colaterais); às características da doença (ausência de sintomas) e ao relacionamento com a equipe de saúde ${ }^{(14-16)}$. Os fatores que interferem na adesão ao medicamento precisam ser conhecidos para que possam ser antecipados e explorados cuidadosamente para que o tratamento seja bem sucedido ${ }^{(7)}$.

Na literatura nacional, não foram identificados estudos sobre a prevalência e fatores relacionados a adesão aos medicamentos entre pacientes com transtorno de ansiedade. Assim, este estudo contribui para preencher uma lacuna no conhecimento sobre essa temática no Brasil.

Considerando-se a hipótese de que há variáveis demográficas, socioeconômicas, clínicas e farmacoterapêuticas envolvidas na adesão ao tratamento, este estudo teve como objetivo verificar, em pessoas com transtorno de ansiedade, possíveis associações entre adesão aos medicamentos e sintomas depressivos, conhecimento sobre a prescrição medicamentosa e perfil demográfico, socioeconômico, clínico e farmacoterapêutico. 


\section{- MÉTODO}

Trata-se de estudo retrospectivo, transversal e descritivo, com abordagem quantitativa. Foi realizado em um serviço ambulatorial de saúde mental, pertencente ao Sistema Único de Saúde, localizado em município do interior do estado de São Paulo - Brasil.

Foi elegível para o estudo a população de pacientes com consulta médica agendada no local de estudo no período de 01 de janeiro a 31 de dezembro de 2012 e que preencheram os seguintes critérios de inclusão: ter diagnóstico pertencente ao grupo dos transtornos de ansiedade (de acordo com a CID-10) ${ }^{(17)}$, estabelecido pelo médico responsável no serviço; ter prescrição de uso contínuo de medicamentos para tratamento do referido transtorno; ter idade igual ou superior a 18 anos e ser capaz de se comunicar verbalmente em português.

Para coletados dados referentes ao perfil demográfico, socioeconômico, clínicoefarmacoterapêutico, foi empregada a técnica de entrevista estruturada, norteada por um questionário desenvolvido pelos autores da presente pesquisa e análise do prontuário do paciente após as entrevistas.

O grau de adesão ao tratamento medicamentoso (variável desfecho) foi avaliado por meio da aplicação do Teste de Medida de Adesão ao Tratamento (MAT). O referido teste foi desenvolvido e validado em Portugal ${ }^{(18)}$ em 2001 e adaptado para o contexto brasileiro ${ }^{(19)}$. É constituído por sete itens, aos quais se responde numa escala tipo Likert, com as respectivas pontuações: Sempre (1), Quase sempre (2), Com frequência (3), Às vezes (4), Raramente (5) e Nunca (6). O nível de adesão é obtido somando-se os valores de cada item e o dividindo pelo número total de itens, correspondendo os valores mais elevados a um maior nível de adesão aos respondentes.

Para identificar a presença de sintomas de ansiedade, foi utilizado o Inventário de Ansiedade de Beck $^{(20)}$, o qual já foi adaptado e validado para o Brasil ${ }^{(21)}$. É um questionário sintomático de auto relato, composto por 21 itens com diferentes alternativas de resposta a respeito de como o sujeito tem se sentido, e que correspondem a diferentes níveis de gravidade da ansiedade. A soma dos escores dos itens individuais fornece um total, que por sua vez constitui um escore dimensional da intensidade da ansiedade, que pode ser classificado nos seguintes níveis: grau mínimo de ansiedade (0-7 pontos), ansiedade leve (8-15 pontos), ansiedade moderada (16-25 pontos) e ansiedade severa (26-63 pontos).

O grau de conhecimento dos participantes sobre os fármacos prescritos foi classificado por meio de um instrumento adotado por Stape ${ }^{(22)}$. Este instrumento indica como traduzir, para porcentagens, a quantidade (números) de informações que o paciente possui e direciona a categorização desse conhecimento. Pressupõe que o grau de conhecimento de uma pessoa sobre cada aspecto relacionado às medicações (nome, dose e frequência de utilização) pode ser pontuado de 0 a $100 \%$ e classificado em intervalos regulares, que representam as seguintes classes: sem conhecimento $(0 \%)$; muito pouco conhecimento ( $0 \%-25 \%)$; pouco conhecimento (25\% -| 50\%); conhecimento regular (50\% - $75 \%$ ) e bom conhecimento $(75 \%-\mid 100 \%)$.

Os participantes foram convidados a participar do estudo por contato telefônico ou na ocasião do comparecimento ao serviço. As entrevistas foram realizadas em salas reservadas, no local de estudo a fim de garantir a privacidade das informações. O tempo médio de duração das entrevistas foi de 30 minutos. Foram explicados os objetivos do estudo e, após o consentimento verbal em participar da pesquisa, foi solicitado que os participantes assinassem o termo de consentimento livre e esclarecido.

Os dados foram digitados em uma base estruturada no formato de planilha, no programa Excel. Posteriormente, foram analisados no programa $S A S^{\circledast}$ 9. Para verificar a associação entre as variáveis independentes e a variável dependente deste estudo, foi utilizado o teste qui-quadrado. O nível de significância adotado neste trabalho foi de $0,05(p<0,05)$.

O estudo foi aprovado pelo Comitê de Ética em Pesquisa sob protocolo 108/2012. 


\section{RESULTADOS}

Dentre os 161 (100\%) participantes da pesquisa, 77,6\% eram do sexo feminino, na faixa etária de 41 a 60 anos (50,3\%), com a cor da pele branca (90,6\%) e com companheiro (62,1\%). As subcategorias de transtorno de ansiedade que se destacaram quantitativamente foram o "transtorno de pânico", o "transtorno misto ansioso e depressivo" (21,1\% cada subcategoria) e o "transtorno de ansiedade generalizada" (20,4\%).

Verificou-se que os antidepressivos foram os medicamentos mais prescritos para os participantes do estudo (91,3\%), seguidos pelos ansiolíticos (69,5\%). Dentre os antidepressivos destaca-se a prescrição de Cloridrato de Sertralina para 61 participantes (37,8\%) e Cloridrato de Fluoxetina para $33(20,4 \%)$. O Teste de Medida de Adesão revelou que a maioria dos participantes era aderente ao tratamento medicamentoso (85\%).

Com o Inventário de Ansiedade de Beck, constatou-se que 50,9\% dos participantes apresentavam grau mínimo de ansiedade. O teste qui-quadrado não revelou diferença estatisticamente significativa entre adesão ao tratamento medicamentoso e sintomas de ansiedade ( $\chi 2=2,93 ; p=0,230)$. Destaca-se, todavia, que houve maior porcentagem de adesão entre pacientes com depressão leve $(92,1 \%)$.

A Tabela 1 apresenta as prevalências de adesão ao tratamento medicamentoso, segundo variáveis demográficas e socioeconômicas dos participantes do estudo.

Não foram encontradas diferenças estatisticamente significativas entre a adesão e as variáveis demográficas e socioeconômicas investigadas, porém, a adesão aos medicamentos foi superior entre os participantes do sexo masculino $(86,1 \%)$, com a cor da pele branca $(86,3)$, sem companheiro $(88,5 \%)$ e desempregados $(100 \%)$.

Observa-se, na Tabela 2, que não houve diferença estatisticamente significativa entre o grau de adesão e as variáveis clínicas investigadas.

Houve maior porcentagem de adesão entre participantes que realizavam apenas o tratamento medicamentoso $(86 \%)$, que tinham comorbidade $(90,6 \%)$, que nunca foram submetidos à internação para tratamento do transtorno de ansiedade $(85,7 \%)$ e que não utilizavam outros tipos de medicamentos $(86,3 \%)$.

Em relação ao conhecimento dos participantes sobre os medicamentos prescritos, houve maior grau conhecimento quanto à frequência de administração dos medicamentos, pois $118(73,2 \%)$ participantes apresentaram "bom conhecimento" por informar corretamente a frequência de administração de 76 a $100 \%$ dos medicamentos prescritos. Constata-se que $55(34,1 \%)$ dos participantes não apresentaram conhecimento sobre a dose dos medicamentos em uso.

Evidencia-se, na Tabela 3, que não houve associação significativa entre a adesão ao tratamento medicamentoso e o grau de conhecimento dos participantes a respeito do nome $(p=0,142)$, da dose $(p=0,448)$ e da frequência de administração $(p=0,295)$ das medicações.

Verificou-se que entre os participantes com grau de conhecimento superior a $50 \%$ em relação a todos os aspectos investigados, houve maior frequência de adesão ao tratamento medicamentoso.

Neste estudo observou-se que a quantidade total de medicamentos $(p=0,649)$, número de comprimidos ao dia $(p=0,752)$, número de vezes que administra a medicação diariamente $(p=0,765)$, compra do medicamento quando em falta na rede pública $(p=0,449)$ e apoio da família $(p=0,532)$ não estiveram associadas à adesão à terapêutica medicamentosa (Tabela 4).

Identificou-se que os sujeitos em monoterapia foram aqueles que apresentaram maior porcentagem de adesão $(89,1 \%)$. Constatou-se maior frequência de adesão ao tratamento medicamentoso entre os participantes que ingerem quantidade igual ou inferior a cinco comprimidos diários (85,3\%), que administram o medicamento uma vez ao dia $(86,2 \%)$, que nunca vivenciaram a falta do medicamento na rede pública $(88,5 \%)$ e que mencionaram o apoio da família $(86,4 \%)$. 
Tabela 1 - Prevalências de adesão ao tratamento medicamentoso, segundo variáveis demográficas e socioeconômicas dos participantes do estudo. Ribeirão Preto, SP, Brasil, 2012

\begin{tabular}{|c|c|c|c|c|}
\hline \multirow{3}{*}{ Variáveis } & \multicolumn{2}{|c|}{ Adesão } & \multicolumn{2}{|r|}{ valor $p$} \\
\hline & Sim & Não & $X^{2^{(1)}}$ & \\
\hline & n (\%) & n (\%) & & \\
\hline Sexo & & & 0,03 & 0,845 \\
\hline Masculino & $31(86,1)$ & $5(13,9)$ & & \\
\hline Feminino & $106(84,8)$ & $19(15,2)$ & & \\
\hline Faixas etárias & & & 0,73 & 0,693 \\
\hline $1^{\circ}$ quarto (19-40 anos) & $34(87,1)$ & $5(12,9)$ & & \\
\hline $2^{\circ}$ quarto (41-60 anos) & $67(82,7)$ & $14(17,3)$ & & \\
\hline $3^{\circ}$ quarto (61-80 anos) & $36(87,8)$ & $5(12,2)$ & & \\
\hline Cor da pele & & & 1,8 & 0,179 \\
\hline Branca & $126(86,3)$ & $20(13,7)$ & & \\
\hline Não branca & $11(73,3)$ & $4(26,7)$ & & \\
\hline Estado civil & & & 0,91 & 0,339 \\
\hline Com companheiro & $83(83)$ & $17(17)$ & & \\
\hline Sem companheiro & $54(88,5)$ & $7(11,5)$ & & \\
\hline Ocupação & & & 0,4 & 0,191 \\
\hline Aposentado & $21(84)$ & $4(16)$ & & \\
\hline Desempregado & $14(100)$ & $0(0)$ & & \\
\hline Trabalhador com vínculo empregatício & $29(85,2)$ & $5(14,8)$ & & \\
\hline Trabalhador autônomo & $18(72)$ & $7(28)$ & & \\
\hline Do lar & $55(87,3)$ & $8(12,7)$ & & \\
\hline Escolaridade & & & 0,06 & 0,97 \\
\hline Sem escolaridade & $33(84,6)$ & $6(15,4)$ & & \\
\hline Fundamental (completo/incompleto) & $55(85,9)$ & $9(14,1)$ & & \\
\hline Médio (completo/incompleto) ou superior & $49(84,4)$ & $9(15,6)$ & & \\
\hline Renda familiar mensal & & & 0,04 & 0,944 \\
\hline Até 3 salários mínimos & $60(85,7)$ & $10(14,3)$ & & \\
\hline Maior ou igual a 3 salários mínimos & $58(85,2)$ & $10(14,8)$ & & \\
\hline
\end{tabular}

\section{- DISCUSSÃO}

Nesse estudo, os medicamentos mais prescritos foram os antidepressivos, seguidos pelos ansiolíticos. Tal resultado é coerente com recomendações da literatura para o tratamento medicamentoso da ansiedade, pois os antidepressivos são os medicamentos de primeira escolha ${ }^{(6)}$.

No entanto, foi identificada alta porcentagem de participantes (69,5\%) em uso de benzodiazepínicos, mesmo após seis meses de início do tratamento com os antidepressivos. Tal fato é preocupante, pois o uso de benzodiazepínicos é contraindicado por tempo prolongado devido à possibilidade de abuso, intoxicação, uso nocivo, dependência e abstinência ${ }^{(5)}$. A prescrição elevada de benzodiazepínicos pode estar associada a conhecimento limitado dos prescritores sobre recomendações de tratamento atuais, falta de resposta aos tratamentos de primeira linha ou preferência do paciente ${ }^{(23)}$.

Este estudo identificou alta porcentagem (85\%) de adesão ao tratamento medicamentoso entre os participantes. A esse respeito, estudo identificou que a maioria dos pacientes abandonou a terapêutica 
Tabela 2 - Prevalências de adesão ao tratamento medicamentoso, segundo variáveis clínicas dos participantes do estudo. Ribeirão Preto, SP, Brasil, 2012

\begin{tabular}{|c|c|c|c|c|}
\hline \multirow{3}{*}{ Variáveis } & \multicolumn{2}{|c|}{ Adesão } & \multirow[b]{2}{*}{$\mathrm{X}^{2^{(1)}}$} & \multirow[b]{2}{*}{ valor $p$} \\
\hline & Sim & Não & & \\
\hline & n (\%) & n (\%) & & \\
\hline Tempo de diagnóstico & & & 0,08 & 0,956 \\
\hline até 5 anos & $49(85,9)$ & $8(14,1)$ & & \\
\hline 6 a 10 anos & $64(84,2)$ & $12(15,8)$ & & \\
\hline 11 anos ou mais & $24(85,7)$ & $04(14,3)$ & & \\
\hline Tipos de tratamento & & & 0,85 & 0,355 \\
\hline Somente com medicamentos & $123(86)$ & $20(14)$ & & \\
\hline Medicamentos + outros & $14(77,7)$ & $04(22,3)$ & & \\
\hline Comorbidades clínicas & & & 1,45 & 0,228 \\
\hline Sim & $39(90,6)$ & $04(9,4)$ & & \\
\hline Não & $98(83)$ & $20(17)$ & & \\
\hline Uso de outras medicações & & & 0,15 & 0,695 \\
\hline Sim & $74(84)$ & $14(16)$ & & \\
\hline Não & $63(86,3)$ & $10(13,7)$ & & \\
\hline Outro transtorno mental & & & 0,03 & 0,861 \\
\hline Sim & $32(84,2)$ & $06(15,8)$ & & \\
\hline Não & $105(85,3)$ & $18(14,7)$ & & \\
\hline Internação para tratamento da ansiedade & & & 0,51 & 0,474 \\
\hline Uma ou mais & $11(78,5)$ & $03(21,5)$ & & \\
\hline Nenhuma & $126(85,7)$ & $21(14,3)$ & & \\
\hline
\end{tabular}

(1) Valor crítico do teste qui-quadrado

Tabela 3 - Prevalências de adesão ao tratamento medicamentoso e grau de conhecimento do nome, dose e frequência de administração dos medicamentos utilizados pelos participantes do estudo. Ribeirão Preto, SP, Brasil, 2012

\begin{tabular}{|c|c|c|c|c|}
\hline \multirow{3}{*}{ Variáveis } & \multicolumn{2}{|c|}{ Adesão } & \multirow[b]{2}{*}{$\mathrm{X}^{2^{(1)}}$} & \multirow[b]{2}{*}{ valor $p$} \\
\hline & Sim & Não & & \\
\hline & n (\%) & $\mathrm{n}(\%)$ & & \\
\hline Grau de conhecimento do(s) nome(s) do(s) medicamento(s) & & & 2,15 & 0,142 \\
\hline $0-0,5$ & $32(78)$ & $9(22)$ & & \\
\hline $0,51-1,0$ & $105(87,5)$ & $15(12,5)$ & & \\
\hline Grau de conhecimento da(s) dose(s) do(s) medicamentos & & & 0,57 & 0,448 \\
\hline $0-0,5$ & $86(83,4)$ & $17(16,6)$ & & \\
\hline $0,51-1,0$ & $51(87,9)$ & $7(12,1)$ & & \\
\hline Grau de conhecimento da frequência do(s) medicamentos & & & 1,09 & 0,295 \\
\hline $0-0,5$ & $27(79,4)$ & $7(20,6)$ & & \\
\hline $0,51-1,0$ & $110(86,6)$ & $17(13,4)$ & & \\
\hline
\end{tabular}

(1) Valor crítico do teste qui-quadrado 
Tabela 4 - Prevalências de adesão ao tratamento medicamentoso e quantidade total de medicamentos utilizados, número de comprimidos ao dia, número de vezes que toma a medicação ao dia, compra o medicamento quando em falta na rede pública e apoio da família. Ribeirão Preto, SP, Brasil, 2012

\begin{tabular}{|c|c|c|c|c|}
\hline \multirow{3}{*}{ Variáveis } & \multicolumn{2}{|c|}{ Adesão } & \multirow[b]{2}{*}{$x^{2^{(1)}}$} & \multirow[b]{2}{*}{ valor $p$} \\
\hline & Sim & Não & & \\
\hline & n (\%) & n (\%) & & \\
\hline Quantidade total de medicamentos & & & 0,86 & 0,649 \\
\hline 1 medicamento & $33(89,1)$ & $4(10,9)$ & & \\
\hline 2 medicamentos & $73(84,8)$ & $13(15,2)$ & & \\
\hline 3 ou mais medicamentos & $31(81,5)$ & $7(18,5)$ & & \\
\hline Número de comprimidos diários & & & 0,09 & 0,752 \\
\hline$<\mathrm{ou}=5$ & $128(85,3)$ & $22(14,7)$ & & \\
\hline 6 a 10 & $9(81,8)$ & $2(18,2)$ & & \\
\hline Número de vezes que toma a medicação ao dia & & & 0,08 & 0,765 \\
\hline $1 \mathrm{vez}$ & $50(86,2)$ & $8(13,8)$ & & \\
\hline 2 vezes ou mais & $87(84,4)$ & $16(15,6)$ & & \\
\hline Compra o medicamento quando em falta na rede pública & & & 1,6 & 0,449 \\
\hline Sim & $57(83,8)$ & $11(16,2)$ & & \\
\hline Não & $18(78,2)$ & $5(21,8)$ & & \\
\hline Nunca faltou na rede & $62(88,5)$ & $8(11,5)$ & & \\
\hline Apoio da família e/ou amigos & & & 0,38 & 0,532 \\
\hline Sim & $89(86,4)$ & $14(13,6)$ & & \\
\hline Não & $48(82,7)$ & $10(17,3)$ & & \\
\hline
\end{tabular}

(1) Valor crítico do teste qui-quadrado

medicamentosa nos primeiros seis meses de tratamento, indicando maior incidência de abandono no início do tratamento ${ }^{(24)}$. Na presente pesquisa todos os participantes estavam em tratamento para o transtorno de ansiedade há mais de seis meses. Uma possível explicação para a alta porcentagem de adesão na população em estudo pode ser a utilização de método indireto para avaliar a adesão, com maior probabilidade de superestimação da adesão ${ }^{(25)}$.

Destaca-se, ainda, que na aplicação do MAT o participante foi questionado sobre a adesão em relação aos medicamentos para o tratamento do transtorno de ansiedade e não, especificamente, em relação aos antidepressivos. Tal informação é relevante ao se considerar que $69,5 \%$ dos entrevistados utilizavam ansiolíticos, podendo ter considerado a adesão a tais medicamentos em suas respostas. A literatura evidencia que há pessoas que têm a percepção de que os benzodiazepínicos produzem efeitos terapêuticos gratificantes e rápidos, favorecendo a adesão ${ }^{(26)}$.

Houve elevada adesão aos medicamentos tanto entre os participantes com sintomas ansiosos leves, como entre aqueles com ansiedade moderada e severa. Em pacientes que aderem ao tratamento medicamentoso, a presença de sintomas ansiosos em intensidade mínima ou leve pode ser explicada, em parte, pela própria adesão. Quanto à alta porcentagem de pacientes com sintomas ansiosos moderados ou severos, é importante considerar a possibilidade de resistência ao tratamento, ou seja, o paciente utiliza o medicamento em doses adequadas, mas não apresenta a resposta desejada, e uma proporção significativa de pacientes não responde aos agentes farmacológicos de primeira linha de forma satisfatória ${ }^{(27)}$.

A resistência ao tratamento medicamentoso afeta cerca de um em cada três pacientes com transtornos de ansiedade. Assim, muitos pacientes permanecem com sintomas residuais clinicamente significativos ${ }^{(28)}$. Estes aspectos reforçam a importância de avaliar sintomas ansiosos, em pacientes em tratamento para transtornos de ansiedade para adequação do esquema terapêutico. Ademais, a inclusão de estratégias psicossociais é relevante para obtenção de melhores resultados ${ }^{(29)}$. 
Embora não tenham sido encontradas diferenças estatisticamente significativas entre a adesão e as variáveis investigadas nesta pesquisa, constatou-se que a adesão ao tratamento medicamentoso foi superior entre participantes do sexo masculino. A relação entre gênero e adesão ainda não foi demonstrada de forma consistente na literatura, todavia há estudos conduzidos com pacientes em uso de antidepressivos e de outros tipos de fármacos que também revelaram maior adesão em pessoas do sexo masculino(30-31).

A esse respeito, estudo que sintetizou dados de revisões sistemáticas sobre a adesão encontrou que o efeito de gênero sobre a adesão não está suficientemente claro, pois são encontrados resultados heterogêneos nas revisões sistemáticas e há falta de explicações consistentes de como o gênero pode influenciar na adesão ${ }^{(32)}$.

A adesão foi superior entre os participantes desempregados. A literatura, todavia, aponta o desemprego como fator de abandono ao tratamento ${ }^{(32-33)}$. Entretanto, são necessários estudos que esclareçam como o emprego pode exercer influencia sobre a adesão, considerando especificidades relacionadas aos tipos de trabalho, ambiente e relações de trabalho ${ }^{(34)}$. Desse modo, sugere-se que estudos futuros sobre a temática considerem tais aspectos, visando elucidar sua influência sobre a adesão à terapia medicamentosa em pessoas com transtorno de ansiedade.

Verificou-se menor porcentagem de adesão aos medicamentos entre os participantes já submetidos a internação psiquiátrica. É importante ressaltar que a internação deveria facilitar a participação ativa do paciente no tratamento, promover a adesão ao tratamento, educação para o autocuidado e realizar planejamento eficaz antes da alta $^{(35)}$.

Em relação ao uso de outras medicações, mostraram-se mais aderentes aqueles participantes que não utilizavam outros tipos de medicamentos (86,3\%). Tal achado é corroborado pela literatura a qual revela que a utilização simultânea de múltiplos fármacos pode desfavorecer o seguimento da terapêutica medicamentosa ${ }^{(36)}$, pois a complexidade do regime terapêutico tem efeito negativo sobre a adesão(32) e não favorece o conhecimento sobre a prescrição medicamentosa pelo paciente.

Destaca-se, nessa pesquisa, a alta porcentagem de participantes com comorbidades clínicas. É importante mencionar a tendência das pessoas com transtorno de ansiedade em buscar auxílio para queixas físicas. Nessas situações é necessário o diagnóstico diferencial e o tratamento adequado tanto para transtornos de ansiedade quanto para comorbidades clínicas ${ }^{(2)}$.

O conhecimento adequado do paciente a respeito dos medicamentos que utiliza constitui fator fundamental para a adesão aos mesmos ${ }^{(37)}$. Nesta pesquisa, entre os participantes com grau de conhecimento superior a 50\% em relação ao nome, dose e frequência de administração dos medicamentos, houve maior frequência de adesão. Chama a atenção a alta porcentagem de participantes com $0 \%$ de conhecimento sobre a dose dos medicamentos em uso. É indispensável que o paciente conheça a dose dos medicamentos que utiliza, tendo em vista as graves consequências que uma dose excessiva de medicamentos pode ocasionar, especialmente em pacientes com transtorno de ansiedade, que recebem prescrição de antidepressivos e ansiolíticos.

Ressalta-se a maior frequência de adesão entre aqueles participantes que mencionaram apoio familiar. As redes de apoio são importantes no enfrentamento de dificuldades, especialmente tratandose de condições crônicas, nas quais é necessário superar as dificuldades prolongadas ${ }^{(38)}$ e a adesão aos medicamentos está ligada à satisfação do paciente com envolvimento dos familiares nos $\operatorname{cuidados}^{(39)}$. Desse modo, a família deve ser incluída e orientada desde o início, a fim de que sua participação no tratamento, no controle de recaídas e na melhora da comunicação possa favorecer a manutenção da estabilidade $^{(40)}$.

\section{CONCLUSÃO}

Nesse estudo, a maioria dos participantes era aderente ao tratamento medicamentoso. A utilização de método indireto para avaliação da adesão pode ter contribuído para tal resultado.

Não houve associação estatisticamente significativa entre a adesão aos medicamentos e as variáveis 
investigadas. Todavia, a maior frequência de adesão foi entre os participantes do sexo masculino, com cor da pele branca, sem companheiro, desempregados, que realizavam apenas o tratamento medicamentoso, com comorbidades, não submetidos à internação para tratamento do transtorno de ansiedade, em monoterapia, que nunca vivenciaram a falta do medicamento na rede pública e que mencionaram o apoio familiar.

Vale ressaltar a alta porcentagem de participantes com ansiedade moderada e severa apesar da adesão aos medicamentos, indicando possível resistência ao tratamento e necessidade de rastreamento desses indivíduos para adequação do esquema medicamentoso e implementação de terapias psicossociais, promovendo a manutenção do tratamento.

Um resultado preocupante identificado no estudo foi a elevada porcentagem de participantes em uso de benzodiazepínicos, mesmo após seis meses de início do tratamento com os antidepressivos, o que contradiz as recomendações atuais para o tratamento medicamentoso de pessoas com transtornos de ansiedade. O uso prolongado de benzodiazepínicos pode acrescentar problemas à pessoa com ansiedade, pois a expõe ao risco de tolerância e dependência. Assim, é necessário que a enfermagem, munida desse conhecimento, assuma um papel ativo na avaliação, orientação e discussão com a equipe e pacientes sobre a prescrição medicamentosa com objetivo de defender as melhores opções de tratamento.

Constatou-se maior frequência de adesão entre os participantes com maior conhecimento sobre seu esquema terapêutico, todavia uma alta porcentagem deles desconhecia a dose dos medicamentos em uso. Esses dados apontam a importância do oferecimento de estratégias educativas que promovam maior segurança e co-participação no tratamento medicamentoso.

Considerando que a adesão aos medicamentos é necessária para o alcance de melhores resultados no tratamento, este estudo traz contribuições ao apontar aspectos relevantes que devem ser sistematicamente investigados e considerados pelos profissionais de saúde na assistência à pessoa com transtorno de ansiedade em tratamento medicamentoso.

\section{REFERÊNCIAS}

1. Combs H, Markman J. Anxiety disorders in primary care. Med Clin North Am. [Internet] 2014; 98(5) [acesso em 05 out 2015]. Disponível: http://dx.doi.org/10.1016/j.mcna.2014.06.003

2. Ramos RT. Transtornos de ansiedade: a prática clínica em doenças reumáticas. Rev Bras Med. [Internet] 2009; 66(11) [acesso em 05 out 2015]. Disponível: http://www.moreirajr.com.br/revistas.asp?fase=r003\&id_materia=4131

3. Pérez-Edgar K, Bar-Haim Y, Mcdermott JM, Gorodetsky E, Hodgkinson CA, Goldman D. Variations in the serotonin-transporter gene are associated with attention bias patterns to positive and negative emotion faces. Biol Psychol. [Internet] 2010; 83(3) [acesso em 05 out 2015]. Disponível: http://dx.doi.org/10.1016/j. biopsycho.2009.08.009

4. Almeida PA, Silva PMC, Espínola LL, de Azevedo EB, Ferreira Filha MO. Desafiando medos: relatos de enfrentamento de usuários com transtornos fóbico-ansiosos. Rev. bras. enferm. [Internet] 2013; 66(4) [acesso em 05 out 2015]. Disponível: http://dx.doi.org/10.1590/S0034-71672013000400010

5. Castro GLG, Mendes CMM, Pedrini ACR, Gaspar DSM, Sousa FCF. Uso de Benzodiazepínicos como automedicação: consequências do uso abusivo, dependência, farmacovigilância e farmacoepidemiologia. R. Interd. [Internet] 2013; 6(1) [acesso em 05 out 2015]. Disponível: http://revistainterdisciplinar.uninovafapi.edu.br/ index.php/revinter/article/view/21

6. Ravindran LN, Stein MB. The pharmacologic treatment of anxiety disorders: a review of progress. J. Clin Psychiatry. [Internet] 2010; 71(7) [acesso em 05 out 2015]. Disponível: http://dx.doi.org/10.4088/JCP.10r06218blu

7. Sansone RA, Sansone LA. Antidepressant adherence: are patients taking their medications? Innov Clin Neurosci. 2012; 9(5-6): 41-6.

8. Martinez IP, López-Torres HJ, Del Campo JMDC, Ferrer VA, Rayo MS, Rabadan EF. Adherence to patients 
antidepressant treatment and the factors associated of non-compiance. Aten Primaria. 2014; 46(7): 357-66.

9. Burton C, Anderson N, Wilde K, Simpson CR. Factors associated with duration of new antidepressant treatment: analysis of a large primary care database. Br J Gen Pract. 2012; 62(595):104-12.

10. Stein MB, Cantrell CR, Sokol MC, Eaddy MT, Shah MB. Antidepressant adherence and medical resource use among managed care patiens with anxiety disoders. Psychiatr Serv. [Internet] 2006; 57(5) [acesso em 22 set 2015]. Disponível: http://psychiatryonline.org/doi/abs/10.1176/appi.ps.57.5.673?url_ver=Z39.88-2003\&rfr_ id=ori:rid:crossref.org\&rfr_dat=cr_pub\%3dpubmed

11. Organización Mundial de la Salud. Adherencia a los tratamientos a largo plazo: pruebas para la acción. Washington; 2004.

12. Freitas JGA, Nielson SEO, Porto CC. Adesão ao tratamento farmacológico em idosos hipertensos: uma revisão integrativa da literatura; Rev Soc Bras Clin Med. 2015; 13(1):75-84.

13. Santana L, Fontenelle LF. A review of studies concerning treatment adherence of patients with anxiety disorders. Patient Prefer Adherence. [Internet] 2011; 5 [acesso em 05 out 2015]. Disponível: http://dx.doi.org/10.2147/PPA. S23439

14. Gardenier D, Andrews CM, Thomas DC, Bookhardt-Murray LJ, Fitzpatrick JJ. Social support and adherence: differences among clients in an AIDS day health care program. J Assoc Nurses AIDS Care. [Internet] 2010; 21(1) [acesso em 05 out 2015]. Disponível: http://dx.doi.org/10.1016/j.jana.2009.06.007

15. Polejack L, Seidl EMF. Monitoramento e avaliação da adesão ao tratamento antirretroviral para HIV+/AIDS: desafios e possibilidades. Cien Saude Colet. [Internet] 2010; 15(1) [acesso em 05 out 2015]. Disponível: http:// dx.doi.org/10.1590/S1413-81232010000700029

16. Hung CL. Factors predicting adherence to antidepressant treatment. Curr Opin Psychiatry. 2014; 27(5):344-9. 17. Organização Mundial da Saúde (OMS). Classificação Estatística Internacional de Doenças e Problemas Relacionados à Saúde - CID 10. 10. ed. São Paulo: Editora da Universidade de São Paulo; 2009.

18. Delgado $A B$, Lima ML. Contributo para validação concorrente de uma medida de adesão aos tratamentos. Psicol. Saúde Doenças. [Internet] 2001; 2(2). [acesso em 05 out 2015]. Disponível: http://repositorio.ispa.pt/ bitstream/10400.12/1114/1/PSD\%202001\%20\%282\%292\%2081-100.pdf

19. Gimenes HT, Zanetti ML, Haas VJ. Factors related to patient adherence to antidiabetic drug therapy. Rev. Latino-Am. Enfermagem. 2009; 17(1):46-51.

20. Beck AT, Epstein N, Brown G, Steer RA. An inventory for measuring clinical anxiety. J. Consult. Clin. Psychol. [Internet] 1988; 56 [acesso em 05 out 2015]. Disponível: http://psycnet.apa.org/journals/ccp/56/6/893.html

21. Cunha J.A. Manual da versão em português das Escalas de Beck. São Paulo: Editora Casa do Psicólogo, 2001.

22. Stape DDB. O conhecimento do paciente com alta hospitalar sobre a continuidade do seu tratamento.

[Dissertação Mestrado]. São Paulo: Escola de Enfermagem da Universidade de São Paulo, 1979.

23. Sorsdahl K, Blanco C, Rae DS, Pincus H, Narrow WE, Suliman S, et al. Treatment of anxiety disorders by psychiatrists from the American Psychiatric Pratice Research Network. Rev. Bras. Psiquiatr. [Internet] 2013; 35(2) [acesso em 05 out 2015]. Disponível: http://dx.doi.org/10.1590/1516-4446-2012-0978

24. Moll MF. O abandono ao tratamento entre pacientes assistidos em um Centro de Atenção Psicossocial. J Nurs Health. [Internet] 2012; 2(1) [acesso em 05 out 2015]. Disponível: http://periodicos.ufpel.edu.br/ojs2/index.php/ enfermagem/article/view/3446/2831

25. Nemes MIB, Helena ETS, Caraciolo JMM, Basso CR. Assessing patient adherence to chronic diseases treatment: differentiating between epidemiological and clinical approaches. Cad. Saúde Pública. [Internet] 2009; 25(3) [acesso em 05 out 2015]. Disponível: http://dx.doi.org/10.1590/S0102-311X2009001500005

26. Lader M. Effectiveness of benzodiazepines: do they work or not? Expert Rev Neurother. [Internet] 2008; 8(8) [acesso em 05 out 2015]. Disponível: http://dx.doi.org/10.1586/14737175.8.8.1189

27. Koen N, Stein DJ. Pharmacotherapy of anxiety disorders: a critical review. Dialogues Clin Neurosci. [Internet] 
28. Menezes GB, Fontenelle LF, Mululo S, Versiani M. Resistência ao tratamento nos transtornos de ansiedade: fobia social, transtorno de ansiedade generalizada e transtorno do pânico. Rev Bras Psiquiatr. [Internet] 2007; 29(2) [acesso em 05 out 2015]. Disponível: http://dx.doi.org/10.1590/S1516-44462007000600004

29. Paulin T, Luzio CA. The psychology in public health: challenges for action and training. Revista de Psicologia da UNESP. [Internet] 2009; 8(2) [acesso em 05 out 2015]. Disponível: http://www2new.assis.unesp.br/index.php/ revista/article/viewArticle/138/171

30. Sawada N, Uchida H, Suzuki T, Watanabe K, Kikuchi T, Handa T, et al. Persistence and compliance to antidepressant treatment in patients with depression: a chart review. BMC Psychiatry. [Internet] 2009; 9(38). [acesso em 05 out 2015]. Disponível: http://dx.doi.org/10.1186/1471-244X-9-38

31. Chen SL, Lee WL, Liang T, Liao IC. Factors associated with gender differences in medication adherence: a longitudinal study. J Adv Nurs. 2014; 70(9):2031-40.

32. Mathes T, Jaschinski T, Pieper D. Adherence influencing factors - a systematic review of systematic reviews. Archives of Public Health. 2014; 72:37.

33. Heck MA, Costa JS, Nunes MF. Tuberculosis treatment drop out prevalence and associated factors in Sapucaia do Sul country (RS). Rev. Bras. Epidemiol. [Internet] 2011; 14(3) [acesso em 05 out 2015]. Disponível: http://dx.doi. org/10.1590/S1415-790X2011000300012

34. International Labour Organization. The Impact of Employment on HIV Treatment Adherence International Labour Office. Geneva, 2013.

35. Castro RA, Aliti GB, Linhares JC, Rabelo ER. Adesão ao tratamento de pacientes com insuficiência cardíaca em um hospital universitário. Rev. Gaúcha Enferm. [Internet] 2010; 31(2). [acesso em 05 out 2015]. Disponível: http:// dx.doi.org/10.1590/S1983-14472010000200004

36. Cruz LP, Miranda PM, Vedana KGG, Miasso AI. Terapêutica medicamentosa: adesão, conhecimento e dificuldades de idosos com transtorno bipolar. Rev. Latinoam. Enferm. [Internet] 2011; 19(4) [acesso em 05 out 2015]. Disponível: http://dx.doi.org/10.1590/S0104-11692011000400013

37. Lee SK, Kang BY, Kim HG, Son YJ. Predictors of medication adherence in elderly patients with chronic diseases using support vector machine models. Healthc. Inform. Res. [Internet] 2013; 19(1) [acesso em 05 out 2015 ]. Disponível: http://dx.doi.org/10.4258/hir.2013.19.1.33

38. Maldaner CR, Beuter M, Brondani CM, Budó MLD, Pauletto MR. Fatores que influenciam a adesão ao tratamento na doença crônica: o doente em terapia hemodialítica. Rev Gaúcha Enferm. [Internet] 2008; 29(4) [acesso em 05 out 2015]. Disponível: http://dx.doi.org/10.1590/S0101-28002012000200002

39. Bolkan CR, Bonner LM, Campbell DG, Lanto A, Zivin K, Chaney E, et al. Family involvement, medication adherence, and depression outcomes among patients in veterans affairs primary care. Psychiatr Serv. 2013; 5:4728.

40. Pellegrinelli KB, Roso MC, Moreno RA. A relação entre a não adesão ao tratamento e falsas crenças de pacientes bipolares e seus familiares. Rev Psiq Clín. [Internet] 2010; 37(4) [acesso em 05 out 2015]. Disponível: http://dx.doi.org/10.1590/S0101-60832010000400009 